\title{
Alguns COMENTÁRIOS SOBRE A QUESTÃO DA IDENTIDADE ENTRE LÓGICAS
}

\author{
Hugo Luiz Mariano ${ }^{1}$
}

\section{Introdução}

O objetivo destas notas, motivadas por palestra proferida no "Lógica no Avião" em setembro de 2016, não é realizar uma discussão conceitual abrangente ou aprofundada sobre o tema da identidade entre entes matemáticos, especificamente no contexto das lógicas, mas apresentar ao leitor interessado um pequeno recorte sobre o tema, focado em alguns encaminhamentos matemáticos, realizados em trabalhos de alguns lógicos, alguns dos quais com atuação ou parcerias acadêmicas no Brasil. Não há qualquer originalidade em abordar o tema sobre identidade/equivalência entre sistemas lógicos, sendo este um assunto recorrente na comunidade dos lógicos; reflexões desta natureza podem ser encontradas, por exemplo, em [5], [10] e [16]. Registramos aqui também que partes do texto [38] foram utilizadas para compor estas presentes notas.

Um primeiro esclarecimento já caberia aqui, originado pela distinção que ocorre na prática entre Lógica como ramo do conhecimento útil para os Fundamentos da Matemática, e a Lógica enquanto área da Matemática com diversas aplicações em outras áreas da própria Matemática (como em Teoria de Modelos). Se por um lado, na primeira concepção da Lógica, é adequado proceder tão mais finitariamente enquanto possível ${ }^{2}$, já no segundo caso, como em Teoria dos Modelos, assumi-se que uma meta-teoria dos conjuntos muito mais rica contendo, por exemplo, ZFC (pelo menos). Nestas notas, por encararem lógicas enquanto objetos matemáticos, vão na segundo direção ${ }^{3}$.

Ilustrando a questão da identidade entre objetos matemáticos:

Consideremos um estudante que entra em contato com a noção matemática denominada grupo, i.e. uma estrutura matemática $(G, \cdot, 1)$, dada por um conjunto (não vazio) $G$, munido de alguns dados: uma operação binária de "multiplicação" (·) que é associativa, e que possui um elemento distinguido (1) que é elemento neutro para esta operação binária, e tal que, além disso, todo elemento admite um (único) inverso (para todo $g \in G$, existe $h \in G$ tal que $g . h=1=h . g$ ), ou seja, a estrutura poderia estar (ou estaria) munida de uma operação unária extra $\left(()^{-1}\right)$ que descreve os inversos multiplicativos de cada elemento. Este estudante não teria dificuldade de aceitar que ambas as apresentações da noção de grupo (com ou sem uma operação unária na linguagem) têm "o mesmo conteúdo matemático", apesar de uma estar dada em linguagem mais completa e satisfazer axiomas mais simples (todos universais) e a outra estar dada em uma linguagem mais restrita e

${ }^{1}$ Instituto de Matemática e Estatística, Universidade de São Paulo. Email: hugomar@ime.usp.br.

${ }^{2}$ Assumindo portanto que na meta-teoria dispomos apenas de uma fraca (meta)teoria de conjuntos (se assim a desejarmos formalizar) que permite ao menos fazermos definições por recursão e provas por indução.

${ }^{3}$ A semântica usual da Lógica de predicados (clássica, finitária) de primeira ordem ou de ordem superior e a própria formulação do que são lógicas infinitárias só podem ser postas no contexto de teorias robustas de conjuntos como ZFC.

DOI : 10.21452/LnA_serie_n_v01_book_seminario-logica-no-aviao-2013-2018_hugo-mariano_p.60-72 
necessitar ser descrita por axiomas mais complexos $(\forall \exists)$. Tecnicamente isto fica respaldado na Lógica de primeira ordem através do "Teorema de Extensão por Definições", cuja formulação pode ser encontrada nos bons livros de Lógica Matemática, e.g. no livro do Shoenfield. Vale acrescer que mesmo esta questão da indistinguibilidade entre teorias matemáticas de primeira ordem admite complexidades e variações não triviais, que envolvem alterações "significativas" de linguagens envolvidas, mas não cabe nestas notas nos determos no detalhamento destas questões ${ }^{4}$.

Entramos agora na questão central destas notas, a indistinguibilidade de lógicas, ilustrando-a inicialmente com pontos específicos:

Via de regra, o primeiro contato de um estudante com lógica simbólica ocorre através de alguma descrição da Lógica Proposicional Clássica e a interpretação dos conectivos básicos $(\wedge, \vee, \neg, \rightarrow)$ por meio de tabelas de verdade a dois valores, $\{0,1\}$, que se estende às interpretações das correspondentes fórmulas proposicionais determinadas por este conjunto de conectivos e um conjunto infinito enumerável de variáveis proposicionais. A este aspecto inicial semântico costuma seguir uma descrição axiomática desta lógica no estilo de Hilbert, i.e. por meio de axiomas e regras de inferência formulados em "parte representativa" desta mesma linguagem. E segue-se usualmente com o estabelecimento de importantes (meta)teoremas, como os metateoremas da Correção e da Completude ${ }^{5}$, que "identificam" essas apresentações semântica e axiomática.

Coloca-se naturalmente neste quadro, a questão semântica dos subconjuntos de conectivos que "determinam a mesma informação" (e.g. $\{\neg, \rightarrow\},\{\neg, \vee\},\{\neg, \wedge\}$ ) ou mesmo quando esta invariância de conteúdo ocorre através de conjuntos que contam com a presença de conectivos alternativos (como o formado apenas pelo símbolo de Sheffer). Há também uma diversidade de descrições axiomáticas alternativas, formuladas em diversas possibilidades de linguagem. Haveria ainda outras alternativas prova-teóricas de capturar o conteúdo dessa lógica além da apresentação axiomática à lá Hilbert, e.g. cálculo de sequentes, dedução natural , tableaux,... Ainda assim, dizemos que estamos nos referindo a mesma lógica proposicional clássica! Mas afinal, o que é, concretamente, a Lógica Proposicional Clássica?([11])

Claro que o problema não se restringe a questão pontual das apresentações da lógica proposicional clássica. A lógica proposicional intuicionista, formulada na linguagem $(\wedge$, $\vee, \neg, \rightarrow$ ) é reconhecida como uma sublógica da lógica clássica (i.e. $\Gamma \vdash_{i} \phi \Rightarrow \Gamma \vdash_{c} \phi$ ) e sendo de fato uma sublógica própria, algo testemunhado, por exemplo, pela ausência do axioma (ou teorema) do terceiro excluído. Reversamente, a toda demonstração clássica está naturalmente associada uma demonstração intuicionista através da "tradução de Glivenko": $\Gamma \vdash_{c} \phi \Rightarrow \neg \neg \Gamma \vdash_{i} \neg \neg \phi$. Nos anos de 1930, Gödel verificou que, diferentemente da lógica clássica, a lógica intuicionista não pode ser caracterizada por uma semântica finita; por outro lado, provou também que o fragmento $(\neg, \wedge)$ da lógica intuicionista "coincide" com a lógica clássica. Assim o problema da identidade de lógicas sugere também

\footnotetext{
${ }^{4}$ Outras noções razoáveis de equivalência entre teorias matemáticas são dadas pelos conceitos de bi-interpretação e interpretação mútua entre teorias: para estas, variantes e outras questões envolvendo aspectos ontológicos de teorias como teorias aritméticas e teorias de conjuntos e classes veja, por exemplo, [3] e [25]. Outras noções de identidades de teorias ainda são possíveis e começaram a ser mais exploradas recementemente, como a noção (categorial) de teorias Morita-equivalentes, que tem sido desenvolvida principalmente por Olivia Caramello.

${ }^{5}$ Este último pode ser estabelecido por pelo menos dois métodos diferentes: há uma abordagem mais efetiva, denominada a prova Kálmar, e outra de cunho algébrico, dada por meio da utilização da álgebra de Boole naturalmente associada à Lógica Clássica, denominada sua Álgebra de Lindenbaum.
} 
a importância e utilidade em se estabelecer critérios mais amplos de comparação entre lógicas.

Tendo em vista os comentário acima, nosso ponto de partida sobre o tema geral da identidade de lógicas é o (aparentemente) mais simples possível, o critério de Leibniz de identidade:

"Objetos $A, A^{\prime}$ são idênticos precisamente quando são indiscerníveis, i.e. A e $A^{\prime}$ compartilham as mesmas propriedades."

Algumas questões surgem imediatamente: O que seria razoável aceitarmos como sendo "propriedades"? Quais seleções de propriedades poderiam estar em jogo? Como nos referirmos aos "dois" objetos $A, A^{\prime}$ que viriam a ser idênticos?

Destas questões, a terceira é mais simples de ser considerada: basta adotarmos o encaminhamento standard de distinguir linguagem objeto e metalinguagem.

Já sobre as duas primeiras questões a situação é muito mais delicada. Um encaminhamento matematicamente simples e natural não seria listar explicitamente o que se pretende aceitar como "propriedades", mas tê-las determinado implicitamente. Mais concretamente: a noção matemática de categoria cumpre bem este papel, consistindo de seleções de uma classe de objetos que são conectados por meio de uma classe selecionada de morfismos, que podem ser compostos de modo a satisfazer leis muito simples, veja por exemplo [32]. O linguajar da Teoria das Categorias fornece imediatamente uma versão simples e razoável da noção de identidade estrutural, dada pelos isomorfismos (= morfismos invertíveis) entre objetos da categoria. Isto já nos leva aos passos seguintes destas notas: considerar categorias cujos objetos "são lógicas". Cabe aqui dizer que, além disso, a Teoria das Categorias também permite estabelecer critérios de comparações de "seleções implícitas de propriedades": por exemplo, as duas apresentações da noção de grupo mencionadas anteriormente são equivalentes pois essas determinam categorias isomorfas.

\section{Motivação principal}

A definição e o estudo sistemático de categorias cujos objetos são lógicas (geralmente proposicionais e com conectivos finitários) foi motivado pelo fenômeno do surgimento de diversos processos de combinações de lógicas que ocorreu na década de 1990, a saber os processos de: produto, fusão, temporalização, sincronização, parametrização e fibrilação (irrestrita e restrita), veja por exemplo [14]. Em geral, estes processos foram definidos em contextos de lógicas particulares (e.g. para lógicas modais) e buscava-se determinar condições que garantissem que fossem preservadas, sob o processo de combinação, certas propriedades meta-lógicas interessantes das lógicas constituintes como as propriedades de: Correção, Completude (ou Completude fraca), Interpolação de Craig, Decidibilidade, etc.

Esses processos de combinação de lógicas se apresentam em aspectos duais: análise de lógicas e síntese de lógicas. Em um processo de combinação, representado aqui por $\otimes$, a relação entre uma lógica $L$ e uma família de lógicas $\left\{L_{i}: i \in I\right\}$, através da "equação" $L \cong \bigotimes_{i \in I} L_{i}$, pode ser encarada de duas formas:

(i) como um processo de decomposição ou análise de lógicas: se a lógica $L$ já foi previamente definida ou considerada, mas está em estudo, e as lógicas da família $\left\{L_{i}: i \in I\right\}$ são bem conhecidas, frequentemente tratando-se de fragmentos mais simples de $L$;

(ii) como um processo de composição ou síntese de lógicas: se as lógicas da família $\left\{L_{i}: i \in I\right\}$ são conhecidas e pretende-se definir (e estudar as meta-propriedades) da 
lógica combinada $L \cong \bigotimes_{i \in I} L_{i}$; ou se as lógicas $\{L\} \cup\left\{L_{i}: i \in I\right\}$ são definidas e conhecidas com a equação significando que foi identificada uma relação entre essas.

Vamos descrever brevemente exemplos típicos de cada um desses aspectos. No início dos anos de 1990, W. Carnielli introduziu o conceito de Semântica de Traduções Possíveis, que baseia-se na idéia de descrever/decompor/analisar uma lógica por meio de uma família de traduções (globalmente conservativa) em lógicas supostamente mais simples ou mais conhecidas que a original ([13]). Dualmente, D. Gabbay, em meados da década de 1990, considerou (originalmente no contexto das lógicas modais e semânticas de Kripke) a noção de fibrilação de lógicas, cujo objetivo seria combinar duas lógicas $L_{1}$ e $L_{2}$ de tal forma a obter "o menor sistema lógico na linguagem combinada que é uma extensão conservativa de ambas as lógicas $L_{1}$ e $L_{2} "([\mathbf{2 7}])$.

Naturalmente, o ponto de partida para tratar categorialmente a questão da combinação de lógicas passa por dois processos de escolha: o de representar os sistemas lógicos que constituem os objetos da categoria em questão e o de descrever os tipos de morfismos entre esses. Dentro da processo de representação dos sistemas lógicos, fixada uma linguagem proposicional (assinatura) e a noção de fórmula correspondente, existem três possibilidades a serem consideradas, com várias alternativas em cada um dessas: (i) a representação sintática das lógicas, dentro do contexto da teoria da prova (sistemas axiomáticos à lá Hilbert, dedução natural, cálculo de sequentes, tableaux, etc); (ii) a representação semântica (valorações, semântica de Kripke, matrizes lógicas, etc); (iii) a representação simultânea ou mista: "lógica $=$ demonstrabilidade + semântica". Fixada a classe de objetos da categoria passa-se à questão da escolha de morfismos conectando esses objetos.

\section{Uma escolha simples}

Frequentemente a maneira mais simples e "concreta" de representar os sistemas lógicos proposicionais é a representação prova-teórica de lógicas como um par $(\Sigma, \vdash)$, onde:

- $\Sigma=\left(\Sigma_{n}\right)_{n \in \mathbb{N}}$ é uma assinatura proposicional e finitária;

- $F_{\Sigma}(X)$ é a $\Sigma$-álgebra completamente livre sobre um conjunto fixado $X$ de "variáveis proposicionais" que é infinito e enumerável;

- $\vdash \subseteq P\left(F_{\Sigma}(X)\right) \times F_{\Sigma}(X)$ é relação de consequência "tarskiana" ([31]) associada a um operador de fecho (das "teorias") $\mathcal{T}: P\left(F_{\Sigma}(X)\right) \rightarrow P\left(F_{\Sigma}(X)\right)$ que é finitário e estrutural (i.e. é compatível com todos os $\Sigma$-homomorfismos de substituição $\left.h: F_{\Sigma}(X) \rightarrow F_{\Sigma}(X)\right)$ : estes operadores sempre são provenientes de algum sistema hilbertiano ${ }^{6}$.

As conexões mais simples e naturais entre os sistemas lógicos como descritos acima são dadas por (alguma variante da noção de) morfismos de tradução (ou interpretação), i.e. certas flechas associadas a funções, definidas nos conjuntos de fórmulas da lógica fonte na lógica alvo, que são "contínuas", i.e. que preservam as relações de consequência envolvidas.

Retornado à motivação principal para considerar categorias de lógicas, observamos que no final dos anos de 1990, o Grupo de Lógica do IST de Lisboa-Portugal e, posteriormente o Grupo de Lógica Teórica e Aplicada do CLE-UNICAMP, consideraram sistematicamente a perspectiva categorial do fenômeno de (certos tipos de) combinações

\footnotetext{
${ }^{6}$ A assinatura e os axiomas e regras de inferência do sistema hilbertiano providenciam uma apresentação da lógica em questão por meio de "geradores e relações" de forma análoga ao que ocorre para as estruturas algébrica dadas por alguma teoria equacional (como as teoria dos grupos, dos anéis, dos reticulados, etc) podem ser apresentadas por geradores e relações.
} 
de lógicas, focalizando atenção em algumas construções categoriais em certas categorias de lógicas, onde procuravam determinar as condições necessárias para que certas metapropriedades fossem conservadas pelas construções. Enquanto o processo analítico que ocorre na Semântica de Traduções Possíveis produz uma tradução conservativa da lógica em estudo em um produto (ou produto fraco) de lógicas mais simples, por outro lado a fibrilação "irrestrita", i.e. sem compartilhamento de conectivos das lógicas constituintes, é descrita pela construção categorial dual de produto, o coproduto, e a noção co-irmã a de fibrilação "restrita", i.e. com compartilhamento de conectivos das lógicas constituintes, é descrito pela construção categorial dual de produto fibrado (ou pullback), a soma amalgamada (ou pushout) ([43], [15]).

A abordagem categorial de noções como a de fibrilação é relevante porque, além desta exigir que os objetos de estudo e suas interrelações sejam totalmente precisos, a caracterização da fibrilação como uma construção universal (como coproduto ou como soma amalgamada) em uma dada escolha de categoria de lógicas, permite a definição, através das mesmas propriedades universais, da noção de fibrilação de lógicas em outras categorias que capturam outros aspectos dos sistemas lógicos: desta forma, foram propostas novas categorias de lógicas que apresentam tratamentos de dois problemas antípodos que ocorreram em certas fibrilações nas primeiras categorias de lógicas: o problema do colapso $([\mathbf{4 2}],([\mathbf{1 8}]))$ e o do anti-colapso $([\mathbf{1 7}])$.

Como exposto acima, a corrente principal do estudo das categorias de lógicas focalizou sua atenção em algumas construções categoriais em certas categorias de lógicas, procurando determinar condições suficientes para que certas meta-propriedades sejam conservadas pelas construções. Posteriormente os aspectos "globais" das categorias de lógicas, que garantem por exemplo a abundância (ou escassez) de construções - algo fundamental para os processos de combinações de lógicas - estabeleceu-se como projeto de investigação complementar ao desta corrente principal. Dentre os trabalhos nesta linha, e que têm em comum essa natureza mais simples de apresentar os sistemas lógicos enquanto objetos, listamos: $[\mathbf{2 2}],[\mathbf{1}],[\mathbf{1 6}],[\mathbf{3 3}],[\mathbf{5}]$.

Em [1], além da representação simples e concreta dos sistemas lógicos por meio de um par formado por uma assinatura e uma relação de consequência tarskiana, tem-se a noção mais simples (porém restrita) e natural possível de morfismo entre assinaturas e de morfismo de tradução induzido entre lógicas: um morfismo (dito estrito) de assinaturas $f: \Sigma \rightarrow \Sigma^{\prime}$ é simplesmente uma sequência de funções que, a cada conectivo $n$-ário da assinatura fonte, $\Sigma$, atribui um conectivo $n$-ário da assinatura alvo, $\Sigma^{\prime}$; este induziria uma função $\hat{f}: F_{\Sigma}(X) \rightarrow F_{\Sigma^{\prime}}(X)$ que, caso preservasse as relações de consequência (i.e. $\left.\Gamma \vdash \phi \Rightarrow \hat{f}[\Gamma] \vdash^{\prime} \hat{f}(\phi)\right)$, determinaria um morfismo (estrito) de lógicas $f:(\Sigma, \vdash) \rightarrow$ $\left(\Sigma^{\prime}, \vdash^{\prime}\right) . \mathcal{S}_{s}$ e $\mathcal{L}_{s}$ denotam as respectivas categorias de assinaturas e de lógicas obtidas através de definições naturais de composição de morfismos e de morfismos de identidades. $\mathcal{S}_{s}$ e $\mathcal{L}_{s}$ têm boas proprieades categoriais pois são completas, co-completas e (finitamente) acessíveis (veja [6] para este conceito): estes resultados constituem o alicerce para outros de resultados apresentados no trabalho em questão.

Como já foi mencionado, uma apresentação de uma lógica como colimite de outras pode ser entendido como uma decomposição da lógica em estudo por meio de outras lógicas (conhecidas e/ou bem comportadas), possivelmente auxiliando no entendimento da lógica mais complexa. Em [1] observou-se que a situação onde uma lógica $L$ é completamente determinada pelas traduções de outras lógicas $L_{i}$ em $L$ pode ser percebida como uma 
"cobertura" de $L$ pelas lógicas $L_{i}$; nesse trabalho transformou-se esta intuição inicial em afirmações matemáticas ao fixar uma categoria de sistemas lógicos e fornecer algumas definições rigorosas da noção de cobertura. Além disso, cada noção de cobertura tomada induz uma topologia de Grothendieck na (essencialmente pequena) subcategoria plena $\left(\mathcal{L}_{s}\right)_{f p} \subseteq \mathcal{L}_{s}$ formada pelas lógicas finitamente presentáveis. Com as noções de feixe assim obtidas foi possível relacionar lógicas (= colimites filtrantes de lógicas finitamente presentáveis) e feixes por intermédio da categoria $\operatorname{Set}^{\left(\left(\mathcal{L}_{s}\right)_{f p}\right)^{o p}}$ dos préfeixes $(=$ colimites de funtores contravariantes hom de lógicas finitamente presentáveis).

Prosseguindo neste ponto de vista global da lógica, estudou-se em [1] uma categoria cujos objetos são as lógicas algebrizáveis no senso de Blok-Pigozzi ${ }^{7}$ (veja [12]). Demonstrouse que a categoria $\mathcal{A}_{s}$, formada pelas lógicas Blok-Pigozzi algebrizáveis com morfismos tomados como os morfismos estritos de lógicas e que também preservam pares algebrizadores (i.e. as equações definidoras e as fórmulas de equivalência da lógica algebrizável), é uma categoria que possui todos os colimites filtrantes, constituindo também uma categoria finitamente acessível. Porém destacamos que esta não é completa, pois não possui limites de diagramas "não limitados", nem cocompleta, pois não possui objeto inicial (nem objeto inicial fraco em geral).

Como mencionamos, foram apresentados em [1] os primeiros passos para se adotar a perspectiva global em Lógica (e se obter informações relevantes a partir desta abordagem) ao tomar escolhas simples e naturais de categorias de assinaturas e de sistemas lógicos. Contudo, na prática, essas escolhas se revelam muito restritivas: tratando de forma inadequada o problema da identidade de sistemas lógicos. Por exemplo, duas apresentações da lógica proposicional clássica que tomam como conjuntos de conectivos primitivos (i.e. assinaturas) $\{\neg, \rightarrow\}$ e $\{\neg, \vee\}$ não admitem nenhum morfismo estrito lógico entre elas (desde que esse precisaria levar $\rightarrow$ em $\vee$ ) enquanto seria natural esperar que essas apresentações fossem isomorfas. Para ir na direção de remediar esse defeito, considerou-se em [22] tomar uma noção mais flexível de morfismo de assinaturas que permitisse levar em conta substitutições nas álgebras de fórmulas, admitindo assim mapear o conectivo binário primitivo $\rightarrow$ no conectivo binário "derivado" $\left(\neg\left(x_{0}\right) \vee x_{1}\right)$ (i.e. uma fórmula em duas variáveis) e onde o conectivo unário $\neg$ seria mapeado em uma fórmula em uma variável $\neg x_{0}$; na direção reversa, também teríamos outro morfismo flexível dado por $\vee \mapsto\left(\neg\left(x_{0}\right) \rightarrow x_{1}\right)$ e $\neg \mapsto \neg x_{0}$. Assim, mapeando-se em geral os conectivos $n$-ários da assinatura fonte do morfismo (dito flexível) em conectivos "derivados" $n$-ários da assinatura alvo (i.e., uma formula da linguagem alvo onde aparecem exatamente as $n$-primeiras variáveis) pode-se obter uma categoria de assinaturas e morfismos flexíveis, $\mathcal{S}_{f}$, e sua correspondente categoria de lógicas e morfismos flexíveis de tradução $\mathcal{L}_{f}$. Contudo, analisando complexidade de fórmulas, é fácil verificar que estes mapeamentos não produziriam isomorfismos inversos de assinaturas ou de lógicas. Além disso, a categoria de assinaturas resultante é bastante

7 Ao passo que o método de algebrização introduzido por Lindenbaum e Tarski de atribuir uma álgebra a uma dada lógica através de uma construção explícita, de modo a fazer um interplay interessante entre a apresentação prova-teórica e a contraparte semântica da lógica em questão, a teoria das lógicas algebrizáveis desenvolvida por Blok e Pigozzi - que, em certo sentido, generaliza aspectos do método anterior - descreve conceitualmente a forma de corresponder sintaxe e semântica (algébrica) em lógicas assim ditas algebrizáveis, através de conjuntos finitos de "fórmulas de equivalência" (fórmulas em duas variáveis) e de um conjunto finito de "equações definidoras" (i.e. pares de fórmulas em uma variável), e tal que ambos os dados detém uma relação estreita com a tal lógica dita algebrizável; em particular existe uma única quasivariedade de álgebras na mesma assinatura da lógica, denominada semântica algébrica equivalente da lógica, na qual se produz uma forma especial de teoremas de correção e completude. 
pobre em termos de construções: e.g. ela não possui pullbacks nem colimites filtrantes. Em [16] foi introduzida uma noção variante de comparação mútua de lógicas através de morfismos flexíveis, $(\Sigma, \vdash) \underset{h}{\stackrel{h^{\prime}}{\rightleftarrows}}\left(\Sigma^{\prime}, \vdash^{\prime}\right)$, denominada equipolência, que pode ser descrita grosso modo dizendo que os morfismos flexíveis $h, h^{\prime}$ são "inversos a menos de interdemonstrabilidade": rapidamente verifica-se que as duas apresentações da lógica clássica dadas acima seriam "equipolentes".

Posteriormente surgiram duas abordagens distintas ([33], [5]), porém em certa medida equivalentes, que refinam os trabalhos mencionados anteriormente ao para lidar simultaneamente, e de forma efetiva, com o problema da identidade de lógicas e a abundância de construções categoriais:

Em [33], foram consideradas categorias de lógicas satisfazendo simultaneamente certos requisitos naturais, tais como:

(i) Representar a maioria dos sistemas lógicos usuais;

(ii) Fornecer um tratamento satisfatório do problema de identidade de sistemas lógicos;

(iii) Possuir boas propriedades categoriais (por exemplo, se elas são categorias completas e/ou cocompletas, se elas são categorias acessíveis);

(iv) Permitir uma noção natural de sistema lógico algebrizável (como no conceito de lógica algebrizável segundo Blok-Pigozzi ([12]) ou de proto-algebrizabilidade de Czelakowski ([19])).

Inicialmente, foram analisadas as duas categorias de assinaturas (finitárias) consideradas na literatura: $\mathcal{S}_{s}$ tem morfismos mais simples mas muito estritos e melhores propriedades categoriais; $\mathcal{S}_{f}$ tem morfismos mais flexíveis mas é categorialmente mais pobre. Definiu-se um par de funtores adjuntos entre essas categorias, mostrando que a mônada ou tripla $\mathcal{T}=(T, \mu, \eta)$ sobre $\mathcal{S}_{s}$, canonicamente associada a esta adjunção, é tal que $T$ preserva colimites filtrantes, reflete isomorfismos e, principalmente, que $\operatorname{Kleisli}(\mathcal{T})=\mathcal{S}_{f}$, de onde se deriva algumas informações adicionais sobre a categoria $\mathcal{S}_{f}$ : por exemplo, esta tem todos coprodutos. Segue-se uma análise da categoria de lógicas $\mathcal{L}_{f}$ associada a categoria de assinaturas $\mathcal{S}_{f}$ : é verificado que a única obstrução para a existência de um "tipo"de limite/colimite em $\mathcal{L}_{f}$ é a não existência deste "tipo" de limite/colimite em $\mathcal{S}_{f}$ : isto já permite concluir que existem em geral (i.e., mesmo no caso infinitário) todas as fibrilações irrestritas e fibrilações restritas quando os $\mathcal{S}_{f}$-morfismos de fonte comum são regulares, i.e. são imagens de $\mathcal{S}_{s}$-morfismos. Define-se a categoria quociente $\mathcal{Q}_{f}:=\mathcal{L}_{f} / \dashv \vdash$, que identifica morfismos flexíveis paralelos entre lógicas cujas imagens de cada fórmula da lógica fonte são inter-demonstráveis na lógica alvo, verifica-se que os $\mathcal{Q}_{f}$-isomorfismos coincidem com as equipolências de [16] e que, portanto, na categoria quociente $\mathcal{Q}_{f}$ diversas apresentações ${ }^{8}$ da lógica clássica são, de fato, isomorfas. Considera-se uma subcategoria plena $\mathcal{L}_{f}^{c} \hookrightarrow \mathcal{L}_{f}$, constituída pelas lógicas ditas "congruenciais" e verifica-se que esta é uma subcategoria reflexiva: logo a toda lógica está canonicamente associada uma lógica congruencial optimal. Finalmente, na correspondente categoria quociente $\mathcal{Q}_{f}^{c}:=\mathcal{L}_{f}^{c} / \dashv \vdash$ : (i) resolve-se o "problema da identidade" para lógica clássica em termos de $\mathcal{Q}_{f}^{c}$-isomorfismos; (ii) a toda lógica esta associada optimalmente um objeto de $\mathcal{Q}_{f}^{c}$; (iii) $\mathcal{Q}_{f}^{c}$ possui boas propriedades categoriais: é completa, cocompleta e finitamente acessível (i.e. toda lógica congruencial é colimite filtrante, em $\mathcal{Q}_{f}^{c}$, de lógicas "finitamente presentáveis"); (iv) existem uma escolha natural de noção de lógica "Lindenbaum" algebrizável, e uma escolha de morfismo

${ }^{8}$ Que não possuem símbolos de constante lógicas como $\top \mathrm{e} \perp$. 
entre essas, de modo a determinar uma categoria $\operatorname{Lind}\left(\mathcal{A}_{f}\right)$ tal que $\operatorname{Lind}\left(\mathcal{A}_{f}\right)=\mathcal{A}_{f} \cap \mathcal{L}_{f}^{c}$ e assim obtendo uma boa subcategoria $\mathcal{Q}\left(\operatorname{Lind}\left(\mathcal{A}_{f}\right)\right) \subseteq \mathcal{Q}_{f}^{c}$.

Em [5], leva-se a campo a teoria abstrata da homotopia aplicada ao contexto das categorias de lógicas. Desta forma tem-se uma "categoria relativa" formada pelo par $\left(\mathcal{L}_{f}, \mathcal{W}\right)$, onde $\mathcal{W}$ é a classe dos morfismos flexíveis que são "equivalências fracas", i.e. as traduções conservativas que são "morfismos densos". Nesta teoria relativa de categorias estão disponíveis as noções de limites e colimites homotópicos, que grosso modo constituem as melhores aproximações de uma construção de limite/colimite que preservam as equivalências fracas. É estabelecido que $\left(\mathcal{L}_{f}, \mathcal{W}\right)$ possui todos os limites e colimites homotópicos. Verifica-se que as equivalências fracas coincidem com as equipolências e que, portanto, a correspondente categoria homotópica (de frações), $\mathcal{L}_{f}\left[\mathcal{W}^{-1}\right]$, é equivalente a categoria quociente $\mathcal{Q}_{f}=\mathcal{L}_{f} / \dashv \models$, situação análoga a que ocorre no contexto da categorias dos espaços topológicos e equivalências de homotopia. Desta forma, esta abordagem não permite apenas uma abundância de construções (homotópicas) e um bom tratamento da questão da identidade de lógicas, mas ela também permite que muitas intuições da teoria da homotopia, desenvolvidas no contexto dos espaços topológicos, sejam transpostas ao contexto das categorias de lógicas.

Finalizamos esta seção observando que mesmo as noções ditas flexíveis de morfismos de traduções de lógicas não englobam a tradução de Glivenko entre a Lógica Clássica e a Lógica Intuicionista. Este ponto sugere que também seria natural considerar ampliações do cenário utilizado nesta seção, tanto na questão da representação de flechas ditas de tradução quanto na representação dos sistemas lógicos como objetos. Desenvolveremos isto na sequência.

\section{Lógicas abstratas e representações}

Nesta seção esboçaremos, com menor nível de detalhes que na seção anterior, alguns desenvolvimentos da apresentação de lógicas e suas conexões que são mais abstratos e/ou dados por meios menos diretos de representação.

Iniciamos observando que, ao invés de se considerar apenas os sistemas lógicos "concretos" como na seção anterior, i.e., aqueles dados por um par $(\Sigma, \vdash)$ onde $\vdash$ está associado a um certo operador de fecho definido na $\Sigma$-álgebra de fórmulas $F_{\Sigma}(X)$, pode ser considerada uma ampliação natural desta idéia, considerando agora uma lógica como dada por um par $(S, \vdash)$ onde $S$ é um conjunto "de sentenças abstratas" e $\vdash: P(S) \rightarrow S$ é um operador de consequência abstrato: este é o ponto de vista desenvolvido em [24], que se concentra no estudo das traduções conservativas abstratas. Alternativamente esta informação pode ser codificada por um par $(S, \mathcal{T})$ dado por conjunto (de "sentenças") munido de um operador de fecho definido em seu conjunto de partes $\mathcal{T}: P(S) \rightarrow P(S)$ : este é o ponto de partida de [41], que apresenta uma variação interessante que incorpora a propriedade de estruturalidade de uma relação de consequência abstrata, trazendo a campo a noção matemática de módulo sobre um quantale para produzir novas noções de identidade de lógicas.

Por um breve momento, vamos utilizar uma situação específica: a da Lógica Clássica de Predicados de primeira ordem - como motivação para algumas abstrações. Aqui cabe reconhermos que a teoria de modelos desta lógica "funciona bem" devido, em grande parte, a dois aspectos: (i) por um lado, em função de (meta)teoremas estruturais específicos 
como o Teorema da Compacidade e o Teorema de Löwenheim-Skolem; (ii) por outro lado, devido a motivos gerais como as construções envolvendo restrições e expansões de linguagem com adições de novos símbolos para constantes e símbolos funcionais. Ambos aspectos admitem generalizações interessantes - a Teoria das Lógicas Abstratas e a Teoria das Instituições- constituindo instâncias de um novo campo de estudos denominado Teoria Abstrata de Modelos, que vamos apresentar brevemente na sequência.

Uma lógica abstrata é uma família de pares ordenados $L=\left(S_{\tau}, \models_{\tau}\right)_{\tau}$ indexada pelas linguagens ou vocabulários $\tau$, onde $S_{\tau}$ é um conjunto ("de $\tau$-sentenças abstratas") e $\models_{\tau}$ é uma relação ("de consequência semântica") entre $\tau$-estruturas e $\tau$-sentenças, satisfazendo algumas condições de compatibilidade. O primeiro Teorema de Lindström caracteriza a Lógica de primeira ordem clássica como a lógica abstrata regular - representando, em particular, os conectivos booleanos - que é maximal entre as que satisfazem a propriedade de compacidade e de Löwenheim-Skolem para baixo: para uma prova deste resultado veja por exemplo o Capítulo XIII de [21]; para variantes deste resultado veja por exemplo $[23]$.

A noção de Instituição foi introduzida por J. Goguen e R. Burstall no final da década de 1970 a fim de providenciar uma unificação formal do conceito de sistema lógico, motivados pela explosão populacional de lógicas e de suas formas de apresentação. Assim este conceito formaliza a noção de sistema lógico como um objeto matemático, i.e., fornece uma teoria de modelo abstrata e categorial que formaliza a noção intuitiva de sistema lógico, incluindo sintaxe, semântica e relação de satisfação entre eles ([20]). A principal característica (modelo-teórica) é que uma instituição contém uma relação de satisfação entre modelos e sentenças (ambos abstratos) que são coerentes sob a mudança de linguagens ou vocabulários (abstratos) por meio de morfismos. Lógicas de predicados de primeira ordem (finitárias ou infinitárias), com a semântica de Tarski, fornecem exemplos naturais de instituições. Uma variação do formalismo das instituições, a noção de $\Pi$-Institutição, foi definida por J. Fiadeiro e A. Sernadas em [26], fornecendo uma abordagem (provateórica) alternativa a noção de instituição ao substituir uniformemente a noção de modelo e satisfação por um operador de consequência primitivo à lá Tarski. Em [37] é verificado que as categorias naturais formadas por lógicas proposicionais, $\mathcal{L}_{s}$ e $\mathcal{L}_{f}$, fornecem exemplos naturais de П-institutições. Neste mesmo trabalho, mostra-se que, com noções naturais de flechas entre instituições e entre П-instituições, obtem-se categorias (grandes), INST e П-INST, e também um par de funtores adjuntos entre estas categorias. Já em [35], é fornecida uma instituição para cada categoria de lógicas proposicionais, $\mathcal{L}_{s}$ e $\mathcal{L}_{f}$, através do uso da noção de matriz para uma lógica proposicional. Neste mesmo trabalho, por uma conveniente modificação da construção anterior, determina-se uma instituição para cada classe de equipolência de lógicas algebrizáveis: isso permite aplicar noções e resultados da Teoria da Instituições em Lógica Proposicional para derivar, a partir da introdução da noção de "contexto de Glivenko", uma forma forte e geral do Teorema de Glivenko, que relaciona um par bem comportado de lógicas proposicionais (como o formado pela lógica clássica e a lógica intuicionista) obtendo assim um par de traduções conservativas entre as lógicas em questão a partir de um morfismo institucional entre as instituições lógicas correspondentes.

Este último resultado indica a utilidade potencial de não apenas considerar versões abstratas de lógicas (sejam proposicionais ou não) mas também considerar formas menos diretas de representar lógicas. Nesta linha aparecem os trabalhos [36], [4] e [34], que descreveremos brevemente na sequência. 
Em [36] são estabelecidos códigos funtoriais perfeitos tanto para morfismos flexíveis de assinaturas quanto para morfismos flexíveis entre lógicas algebrizáveis. Em mais detalhe: (i) é estabelecido um anti-isomorfismo entre a classe de morfismos de assinaturas e alguns funtores entre as respectivas categorias de estruturas; (ii) prova-se que este antiisomorfismo se restringe a um anti-isomorfismo entre morfismos de lógicas algebrizáveis e alguns funtores entre suas categorias de estruturas que se restringem às suas quasivariedades canônicas que são suas respectivas semânticas algébricas equivalentes.

A lacuna conceitual do trabalho [36]- a saber a ausência de um código funtorial perfeito para lógicas proposicionais gerais - é preenchida pelo trabalho [4]. Isto é realizado através da noção de par filtro, um conceito introduzido em [39], como uma ferramenta para criar e analisar lógicas. Em mais detalhes: um par filtro para uma assinatura $\Sigma$ é um par $(G, j)$ dado por funtor contravariante $G$ da categoria das $\Sigma$-estruturas na categoria dos reticulados algébricos e por uma transformação natural $j: G \rightarrow P$, onde $P: \Sigma-S t r \rightarrow A L$ é o funtor contravariante dado pelo conjunto potência do conjunto subjacente a uma estrutura, e tal que as componentes de $j$ são funções que preservam ínfimos arbitrários e supremos dirigidos. É mostrado que toda lógica tarskiana surge de um par filtro e que as traduções flexíveis de lógicas surgem de morfismos de pares filtros, estabelecendo uma conexão categorial precisa: a categoria $\mathcal{L}_{f}$ é isomorfa a uma subcategoria plena e coreflexiva da categoria formada por pares filtros e morfismos adequados de "mudança de assinatura base".

Os trabalhos mencionados nos dois parágrafos anteriores apresentam um traço comum: produzir códigos (funtoriais) perfeitos de lógicas. Em Matemática, existem várias instâncias úteis de codificações mais relaxadas, como por exemplo quando se representam grupos por grupos de matrizes ou quando se identificam anéis com unidade $R, R^{\prime}$ que são Morita-equivalentes, i.e., quando as respectivas categorias de representações lineares (categorias de módulos) são equivalentes: $R-M o d \simeq R^{\prime}-M o d$. Esta última relação está longe de constituir uma representação fiel da identidade estrutural de anéis ${ }^{9}$ e daí segue sua utilidade. Em [34] são dados os primeiros passos para se constituir uma teoria de representações de lógicas: (i) seleciona-se uma classe de objetos padrões "bem comportados", a classe das lógicas (Lindenbaum) algebrizáveis, que têm uma boa representação categorial (dada pelas quasivariedades canonicamente associada a essas); (ii) por meio desta classe de objetos especiais define-se o conceito de lógicas Morita equivalentes (à esquerda) e uma variante mais fraca denominada lógicas estavelmente Morita equivalentes (à esquerda); (iii) mostra-se que uma lógica e sua lógica congruencial canonicamente associada são Morita equivalentes (apesar de, em geral, não serem equipolentes); (iv) mostra-se que as diversas apresentações da lógica clássica são Morita equivalentes; (v) verifica-se que a lógica clássica e a lógica intuicionista não são estavelmente Morita equivalentes.

Finalizamos estas notas com algumas palavras sobre identidades de ordem alta entre lógicas. Anteriormente nos focamos nas apresentações (e representações) de sistemas lógicos sob um ponto de vista prova-teórico, e também o mais básico entre esses: baseadas essencialmente na apresentação (ou codificação) de apenas uma relação de demonstrabilidade, eventualmente abstrata. Na realidade esse está longe de ser o espírito principal da subárea da Lógica denominada "Teoria da Prova", que como o próprio nome coloca, foca

\footnotetext{
${ }^{9}$ I.e., existem anéis Morita equivalentes que não são isomorfos. Por exemplo, os anéis $R$ e $M a t_{n \times n}(R)$ são sempre Morita equivalentes, para qualquer $n \geqslant 1$, mas mesmo que $R$ seja comutativo, o anel $\operatorname{Mat}_{n \times n}(R)$ nunca é comutativo se $n \geqslant 2$, logo estes não podem ser anéis isomorfos.
} 
seu estudo nas provas em si e não na relação de demonstrabilidade (i.e. se existe uma prova de uma fórmula (eventualmente abstrata) a partir de algum conjunto de fórmulas). Assim, por esse ponto de vista, seria muito natural considerar identidades entre provas como parte constituinte de alguma conceituação de identificação de lógicas: por exemplo, colocando que duas provas são indistinguíveis quando se reduzem à mesma forma normal. A situação seria, grosso modo, assim descrita: a cada lógica atribuímos uma multi-categoria ([30]) cujos objetos são as fórmulas e cujas multi-flechas, que tem fonte um conjunto de fórmulas e alvo uma fórmula única, são as provas desta fórmula com hipóteses no conjunto; a composição de multi-flechas seria dada pela concatenação de provas (como esperado); as multi-identidades seriam dadas pela propriedade de reflexividade ${ }^{10}$. Assim os sistemas lógicos anteriormente considerados seriam simplesmente "multi-posets", uma versão "magra" da multi-categoria acima descrita: i.e. haveria uma única multi-flecha de um conjunto de fórmulas em uma fórmula sempre que existisse alguma prova da fórmula com hipóteses neste conjunto. Assim, sob o ponto de vista anteriormente discutido, lógicas seriam indistinguíveis quando os multi-posets associados fossem equivalentes. Por outro lado, na multi-categoria cheia, cujas multi-flechas são as provas, haveria muito mais estrutura a ser considerada, tendo assim noções muito mais finas de indistinguibilidade de lógicas: lógicas seriam indistinguíveis se, além de haver um processo de identificação dos objetos dados por (classes de equivalência) de fórmulas, este processo também respeitasse (e identificasse) a estrutura local de provas entre as fórmulas associadas.

Tendo em vista as colocações acima e trazendo também a campo a essência da correspondência de Curry-Howard, que conecta lógicas com teorias de tipos - identificando fórmulas com tipos e assim também identificando provas de fórmulas com termos de um dado tipo - fica natural entendermos as iniciativas que surgiram a partir de meados da década de 1990 de conectar teoria de tipos e teoria da homotopia abstrata $([\mathbf{2 9}])$. Assim a questão de identidades entre provas ficaria codificada através da família, indexada nos tipos, formada pelos tipos de identidade associado a cada tipo, que codifica a estrutura das noções de indistinguibilidade entre termos de um dado tipo. Assim as próprias identidades entre termos (provas) de um dado tipo (fórmula) seriam dados por "homotopias formais" entre esses termos. Por outro lado, estas homotopias formais poderiam ser realizados concretamente por caminhos computacionais (reversíveis) entre provas (= multi-flechas) dados por sequências finitas de substituições e reescrituras que transformariam uma prova na outra prova. E também poderíamos imaginar identidades de ordem ainda mais alta, por exemplo, observando a estrutura das homotopias formais entre homotopias formais, e assim por diante.

No espírito das observações colocadas nos parágrafos acima, finalizamos essas notas simplesmente sugerimos ao leitor interessado a consulta das referências $[\mathbf{2 9}],[\mathbf{2 8}],[\mathbf{2}]$ e $[40]$.

10 Toda fórmula determina uma prova dela mesma. 


\section{Referências Bibliográficas}

[1] P. Arndt, R. A. Freire, O. O. Luciano, H. L. Mariano, A global glance on categories in Logic, Logica Universalis 1 (2007), 3-39.

[2] P. Arndt, C. Kapulkin, Homotopy Theoretic Models of Type Theory, arXiv preprint, https://arxiv.org/abs/1208.5683, 2012.

[3] E. L. B. de Almeida, Análise das condições de verdade e dos requerimentos existenciais em axiomatizações da aritmética, Tese de Doutorado em Filosofia, IFCH-Unicamp, 2017.

[4] P. Arndt, H. L. Mariano, D. C. Pinto, Finitary Filter Pairs and Propositional Logics, a aparecer em South American Journal of Logic, 2019.

[5] P. Arndt, Homotopical Categories of Logics, em The Road to Universal Logic (2015), 13-58.

[6] J. Adámek, J. Rosický, Locally Presentable and Accessible Categories, Lecture Notes Series 189, London Math. Soc., Cambridge University Press, 1994.

[7] J. Bueno-Soler, W. A. Carnielli, Possible-translations algebraization for paraconsistent logics, Bulletin of the Section of Logic, University of Lodz, Poland, vol. 34, n. 2, 2005, pp. 77-92. CLE e-Prints vol.5 n.6, (2005), http://www.cle.unicamp.br/e-prints.

[8] J. Bueno, M. E. Coniglio, W. A. Carnielli, Finite algebraizability via possible-translations semantics, Proceedings of CombLog'04 — Workshop on Combination of Logics: Theory and Applications, Departamento de Matemática, Instituto Superior Técnico, Lisboa, Portugal, 2004.

[9] J. Bueno-Soler, M. E. Coniglio, W. A. Carnielli, Possible-Translations Algebraizability, Paraconsistency with no Frontiers (J.-Y. Béziau, W. Carnielli, eds.), North-Holland, 2006.

[10] J.-Y. Béziau, From Consequence Operator to Universal Logic: A Survey of General Abstract Logic, em Logica Universalis: Towards a General Theory of Logic (J.-Y. Béziau, ed.), Birkhäuser, 2007, 3-17.

[11] J.-Y. Béziau, R. P. de Freitas, J. P. Viana, What is Classical Propositional Logic? (A Study in Universal Logic), Logica Studies 7, 2001.

[12] W. J. Blok, D. Pigozzi, Algebraizable logics, Memoirs of the AMS 396, American Mathematical Society, Providence, USA, 1989.

[13] W. A. Carnielli, Many-valued logics and plausible reasoning, Proceedings of the XX International Congress on Many-Valued Logics, IEEE Computer Society, University of Charlotte, USA, 1990, 328-335.

[14] W. A. Carnielli, M. E. Coniglio, Combining Logics, Stanford Encyclopedia of Philosophy, http://plato.stanford.edu/entries/logic-combining/, 2016.

[15] C. Caleiro, W. Carnielli, J. Rasga, C. Sernadas, Fibring of Logics as a Universal Construction, Handbook of Philosophical Logic 13 (editores: D. Gabbay, F. Guenthner), Kluwer Academic Publishers, 2005.

[16] C. Caleiro, R. Gonçalves, Equipollent logical systems, em Logica Universalis: Towards a General Theory of Logic (J.-Y. Béziau ed.), 2007, 97-110.

[17] M. E. Coniglio, The Meta-Fibring environment: Preservation of meta-properties by fibring, CLE e-Prints 5(4) (2005).

[18] C. Caleiro, J. Ramos, Cryptofibring, Proceedings of CombLog 04 - Workshop on Combination of Logics: Theory and Applications, Lisboa, Portugal (editores: W. A. Carnielli, F. M. Dionísio, P. Mateus), 2004, 87-92.

[19] J. Czelakowski, Protoalgebraic logic, Trends in Logic, Studia Logica Library, Kluwer Academic Publishers, 2001.

[20] R. Diaconescu, Institution-independent Model Theory, Birkhäuser, 2000.

[21] H. D. Ebbinghaus, J. Flum, W. Thomas, Mathematical Logic, Undergraduate Texts in Mathematics, segunda edição, Springer-Verlarg, 1994. 
[22] V. L. Fernández, M. E. Coniglio, Fibring algebraizable consequence systems, Proceedings of CombLog 04 - Workshop on Combination of Logics: Theory and Applications, (editores: W. A. Carnielli, F. M. Dionísio, P. Mateus), 2004, 93-98.

[23] J. Flum, Characterizing Logics, Capítulo 2 em Model-Theoretic Logics (ed. J. Barwise, S. Feferman), Perspectives in Mathematical Logic, Springer-Verlag, 1985.

[24] H. A. Feitosa, I. M. L. D’Ottaviano, Conservative translations, Annals of Pure and Applied Logic 108 (2001), 205-227.

[25] A. R. de O. Freire Filho, Estudo Comparado do Comprometimento Ontológico das Teorias de Classes e Conjuntos, Tese de Doutorado em Filosofia, IFCH-Unicamp, 2019.

[26] J. Fiadeiro, A. Sernadas, Structuring theories on consequence, Recent Trends in Data Type Specification (D. Sannella, A. Tarlecki eds.), Lecture Notes in Comput. Sci. 332 (1988), 44-72.

[27] D. Gabbay, Fibred semantics and the weaving of logics: Part 1, Journal of Symbolic Logic 61(4) (1996), 1057-1120.

[28] Homotopy Type Theory: Univalent Foundations of Mathematics, Institute for Advanced Study, disponível em https://homotopytypetheory.org/book/.

[29] M. Hofmann, T. Streicher, The groupoid interpretation of type Theory, em Twenty-Five Years of Constructive Type Theory (Venice, 1995), Oxford Logic Guides 36, Oxford University Press, 1998, 83-111.

[30] T. Leinster, Higher operads, higher categories, Lecture Notes Series 298, London Math. Soc., Cambridge University Press, 2004.

[31] J. Łoś, R. Suszko, Remarks on sentential logics, Proceedings Koninkliske Nederlandse Akademie van Wetenschappen, Series A, 61 (1958), 177-183.

[32] S. MacLane, Categories for the Working Mathematician, Graduate Texts in Mathematics 5, segunda edição, Springer-Verlag, 1998.

[33] H. L. Mariano, C. A. Mendes, Towards a good notion of categories of logics, arXiv preprint, http://arxiv.org/abs/1404.3780, 2016.

[34] H. L. Mariano, D. C. Pinto, Representation theory of logics: a categorial approach, arXiv preprint, http://arxiv.org/abs/1405.2429, 2014.

[35] H. L. Mariano, D. C. Pinto, An abstract approach to Glivenko's theorem, arXiv preprint, http://arxiv.org/abs/1612.03410, 2016.

[36] H. L. Mariano, D. C. Pinto, Algebraizable Logics and a functorial encoding of its morphisms, Logic Journal of the IGPL 25(4) (2017), 524-561.

[37] H. L. Mariano, D. C. Pinto, Remarks on Propositional Logics and the Categorial Relationship Between Institutions and П-Institutionst, South American Journal of Logic 3(1) (2017), 111-121.

[38] H. L. Mariano, Grupos Especiais, Teoria dos Modelos e Categorias de Lógicas, Tese de Livre-Docência, IME-USP, 2011.

[39] D. C. Pinto, A categorial foundation for a representation theory of logics, Tese de Doutorado em Matemática, IME-USP, 2016.

[40] R. J. G. B. de Queiroz, A. G. de Oliveira, A. F. Ramos, Propositional Equality, Identity Types, and Computational Paths, South American Journal of Logic 2(2) (2016), 245-296.

[41] C. Russo, An order-theoretic analysis of interpretations among propositional deductive systems, Annals of Pure and Applied Logic 164 (2013), 112-130.

[42] C. Sernadas, J. Rasga, W. A. Carnielli, Modulated fibring and the collapsing problem, The Journal of Symbolic Logic 67 (2002), 1541-1569.

[43] A. Sernadas, C. Sernadas, C. Caleiro, Fibring of logics as a categorial construction, Journal of Logic and Computation 9(2) (1999), 149-179. 